\title{
UNE INQUIÉTANTE FRACTURE DISCURSIVE
}

\author{
Dominique Maingueneau $^{1}$
}

RÉSUMÉ: On voit se développer des phénomènes qu’on désigne diversement : « montée du populisme ", "mouvements antisystèmes ", etc. Ce n'est pas seulement une fracture sociale, mais aussi une fracture "discursive ". Les " populistes " pensent qu'on leur refuse l'accès aux lieux où s'énoncent les paroles qui ont le plus de poids en termes d'audience et d'autorité morale, qu'une élite de privilégiés et/ou de minorités coupée de la société " réelle » a le monopole de la parole légitime. Cette fracture discursive est rendue possible par les réseaux sociaux, où ceux qui se pensent exclus s'expriment abondamment. Mais il existe une asymétrie fondamentale entre leurs énoncés et ceux des " élites ", qui justifient leur statut en respectant un certain nombre de normes cognitives et linguistiques. C'est une dangereuse situation d' « interincompréhension » où chacun des deux adversaires se légitime par l'autre. L'analyste du discours ne peut pas se contenter de montrer les déficiences des énoncés " populistes »; il doit réfléchir sur la fracture discursive elle-même. Mais traiter le discours des élites comme du discours ne doit pas signifier valider le point de vue "populiste».

MOTS-CLÉS : Fracture discursive. Mouvements antisystèmes. Montée du populisme. Bolsonaro. Trump. Réseaux sociaux.

\section{UMA FRATURA DISCURSIVA PREOCUPANTE}

RESUMO: Acompanhamos o desenvolvimento de fenômenos que designamos de várias maneiras: "ascensão do populismo", "movimentos antissistema" etc. Não se trata, apenas, de uma fratura social, mas, também, de uma fratura discursiva. Os populistas consideram que a eles foi impedido o acesso ao lugar de fala nos ambientes em que há maior audiência ou

\footnotetext{
1 Universidade Paris IV-Sorbonne, Departamento de Língua Francesa, Paris - França. Professor de Linguística. Orcid : http://orcid.org/0000-0001-8907-218X. dominique.maingueneau@sorbonne-universite.fr.
} 
autoridade moral / credibilidade, que uma elite de privilegiados e / ou minorias distanciadas da sociedade "real" têm o monopólio da legitimidade da fala. Essa fratura discursiva se tornou possivel por meio das redes sociais, em que aqueles que se sentem excluidos podem falar o que querem. Existe, contudo, uma assimetria fundamental entre esses discursos e os das "elites", as quais justificam seu estatuto respeitando um certo número de normas cognitivas e linguisticas. É uma perigosa situação de "interincompreensão", em que cada um dos dois adversários se legitima pelo outro. $O$ analista do discurso, portanto, náo pode se contentar em mostrar as deficiências dos discursos "populistas"; ele deve refletir sobre a própria fratura discursiva. Tratar a fala "populista" como discurso, contudo, não deve significar a validação de seu ponto de vista.

PALAVRAS-CHAVE: Fratura discursiva. Movimentos antissistema. Ascensão do populismo. Bolsonaro. Trump. Redes sociais.

\section{A WORRYING DISCURSIVE FRACTURE}

ABSTRACT: We face the development of a variously designated phenomena: "rise of populism", "anti-system movements", etc. It is not only a social fracture, but also a "discursive" one. The populists think that they have been denied from accessing the right to the speech in environments in which there is a greater audience or moral authority / credibility, that an elite of privileged and / or minorities separated from "real" society have a monopoly on the legitimacy of speech. This discursive fracture is possible by social networks, in which those who think they are excluded express themselves abundantly. But there is a fundamental asymmetry between their statements and those of the "elites", which justify their status by respecting a certain number of cognitive and linguistic norms. It is a dangerous situation of "interincomprehension" in which each of the two adversaries legitimizes itself by the other. Discourse analysts cannot be content with showing the deficiencies of "populist" statements; they must reflect on the discursive fracture itself. But treating the speech of the elites as discourse must not mean validating the "populist" point of view.

KEYWORDS: Discursive fracture. Antisystem movements. Rise of populism. Bolsonaro. Trump. Social network.

Depuis un certain nombre d'années on voit se développer dans de nombreux pays des phénomènes qu’on désigne de diverses manières : " montée du populisme ", " affaiblissement des grands partis ", " crise de la représentation ", 
" rejet des élites ", " mouvements antisystèmes ", etc. La diversité de ces dénominations témoigne de la perplexité dans laquelle se trouvent les spécialistes, chacun mettant l'accent sur telle ou telle facette de phénomènes qu'il est très difficile de cerner. Le problème est qu'il ne s'agit pas seulement d'agitations à la périphérie des fonctionnements politiques classiques, mais que cela a permis ou risque de permettre à certaines personnalités inquiétantes d'accéder au pouvoir.

Face à cela, les analystes du discours ont spontanément une réaction de rejet. Parce que de tels phénomènes vont à l'encontre des convictions personnelles de la grande majorité d'entre eux ; mais aussi parce que la pratique même des sciences sociales s'oppose aux catégorisations et aux raisonnements simplistes, à la désignation de boucs émissaires, à la diffusion de fausses rumeurs, à la révélation de complots, à l'expression de la haine..., bref à tout ce qu'ils jugent caractéristique de ces discours. Quand ils en font un objet d'étude, ils nourrissent l'espoir que leurs recherches, portées par une volonté critique, vont contribuer à les discréditer. À vrai dire, cette entreprise ne peut que réussir : il n'est pas difficile de mettre au jour dans ces corpus une multitude de déviances cognitives ou morales, qui contrastent avec le sérieux des méthodes qui les analysent. Même si c'est à travers des concepts et des procédures beaucoup plus élaborés, les analystes du discours procèdent de la même manière que les journaux destinés aux élites qui dans le monde entier s'attachent à corriger systématiquement les fake news, à dissiper ce qu'ils considèrent comme des approximations ou des mensonges au service de forces négatives : haine, ethnocentrisme, fanatisme, machisme...

Pour exercer pleinement une fonction critique, l'analyse du discours doit être à la mesure des objets qu'elle étudie, éviter de leur appliquer des catégories et des procédures qui ont été conçus pour des corpus d'un type différent. Les objets que nous étudions sont inscrits dans l'histoire et nous ne pouvons jamais être assurés a priori que la boîte à outils dont nous disposons est pertinente partout et en tous temps. Une des caractéristiques du monde actuel est la globalisation associée à la transformation brutale et généralisée des technologies de la communication. Pour un analyste du discours cela ne peut qu'avoir des incidences profondes sur le discours et la nature des acteurs politiques. On sait par exemple que les réseaux sociaux sont un terrain privilégié de manipulation de l'opinion par divers types d'agents ou que certains gouvernants utilisent Twitter pour communiquer directement avec les citoyens, mettant ainsi hors jeu les médiateurs institutionnels. Cela implique que les analystes du discours adaptent leur outillage en conséquence s'ils ne veulent pas passer à côté de leur objet.

En matière d'analyse du discours politique la recherche vise traditionnellement à comparer les discours de groupes qui occupent une position bien définie 
dans le champ politique ; or les phénomènes qui nous intéressent ici semblent résister à cette logique de positionnement classique, structurée par des partis qui sont en compétition pour emporter l'adhésion d'électeurs. Certes, cette logique continue à fonctionner, mais elle est minée et fragilisée par ce que j’appellerai une "fracture discursive". Non pas une fracture à l'intérieur d'un discours, mais de l'espace public lui-même, fissuré par la divergence entre deux régimes hétéronomes du discours. Cette fracture est sans doute liée à la globalisation et aux transformations des modes de communication, mais elle s'explique aussi par d'autres facteurs, qui interagissent avec les deux premiers.

Les «Gilets jaunes" français ne sont pas les électeurs de J. Bolsonaro ou de D. Trump, mais beaucoup d'entre eux partagent quelque chose qui ne peut manquer d'attirer l'attention des analystes du discours : ils ne dénoncent pas seulement les privilèges, la corruption, le mépris ou l'indifférence des élites à leur égard, etc., comme il est de tradition dans les mouvements dits "populistes ", mais ils contestent une certaine distribution du discours qui leur refuse l'accès à ce qu'on peut appeler la "Sphère autorisée ", c'est-à-dire les lieux où s'énoncent les paroles qui ont le plus de poids, qui comptent en termes d'audience et d'autorité morale : d'une part les stations de radio, les chaînes de télévision, les journaux et les magazines les plus importants, ainsi que les sites web qui leur sont liés, d'autre part les institutions officielles (politique, justice, éducation...). Cette Sphère est perçue comme étant au service d'une élite de privilégiés et/ou de minorités coupée de la société « réelle » qui se seraient illégitimement approprié le pouvoir de décider ce qu'il est permis et ce qu'il est interdit de dire, disqualifiant ainsi les hommes et les femmes "de bon sens", " ordinaires ", les "petites gens "... On ne leur donne pas la parole ou, quand on le fait, ils sont présentés comme des déviants et traités comme tels.

Ici intervient la question du " politiquement correct ». Cette police du langage n'est activement soutenue que par très peu de gens, mais pour ceux qui dénoncent les élites elle constitue la version la plus radicale de cette police du discours qui disqualifie leurs paroles. Le politiquement correct présente trois caractéristiques intéressantes : 1) ce n'est pas une doctrine politique, même s'il s'appuie indirectement sur certaines forces politiques; il est plutôt lié à une multitude d'organisations très diverses : féministes, homosexuelles, antiracistes, écologistes, antialcooliques, anticolonialistes...;2) il a une teneur essentiellement morale ; autrement dit, ceux qui s'y opposent sont appréhendés beaucoup moins comme des adversaires politiques que comme des hommes ou des femmes à l'éthique déviante ; 3) il s'efforce de contrôler le langage, à deux niveaux : celui du système de la langue, et celui du discours. Les militants les plus convaincus 
entendent transformer la langue elle-même (en particulier certains aspects de la morphologie, du lexique et de l'orthographe), tandis que d'autres, beaucoup plus nombreux, s'efforcent seulement, quand ils parlent, de ne pas dévier d'une orthodoxie implicite, d'éviter ce qui (des mots, des thèmes, des points de vue, des argumentations...) pourrait susciter un lynchage médiatique, les mettre en marge de la communauté morale à laquelle ils pensent devoir appartenir pour parler avec autorité. Cette attitude présuppose que la parole doit être régie par des normes intellectuelles et des valeurs supérieures, et que ceux qui les rejettent énoncent des paroles en quelque sorte sauvages, à la fois intellectuellement déficientes et au service d'intérêts égoïstes.

La fracture discursive s'organise autour d'une opposition élémentaire et fortement axiologisée entre le Haut et le Bas. Mais la valeur qui est conférée à ce Haut et ce Bas n'est pas la même dans les deux cas. Pour les uns, la position haute tient à la supériorité morale et intellectuelle des valeurs dont ils se réclament, à leur effort pour résister à des forces venues d'en bas ; pour les autres, la parole vraie est au contraire celle d'en bas, du peuple, et la position haute que revendiquent ceux qui les disqualifient est une oppression illégitime, un déni de démocratie.

On se heurte alors à une difficulté : comment désigner les locuteurs de l'un et l'autre régime sans adopter le point de vue de l'un d'eux ? La solution la plus commode consiste à utiliser des dénominations distinctes, selon qu'on se place dans un camp ou dans l'autre.

Point de vue et type de « locuteurs"

\begin{tabular}{|l|l|l|}
\hline & Evalué positivement & Evalué négativement \\
\hline Point de vue-M (Moral) & Locuteur-M (Moral) & Locuteur-IM (Immoral) \\
\hline Point de vue-P (Populaire) & Locuteur-P (Populaire) & Locuteur-EG (Elites globalisées) \\
\hline
\end{tabular}

Source: Propre organisation.

J'ai distingué ici quatre types de "locuteurs ", et non de personnes, d'acteurs ou d'individus. Il est permis de penser - même si cela complique considérablement les choses - que la fracture discursive passe à l'intérieur même d'un certain nombre d'individus qui alimentent, en fonction des situations de parole, les deux régimes, probablement sans trop s'en rendre compte. Seule une minorité défend en toutes circonstances le même point de vue.

Le terme « Elites » dans « Elites globalisées » n’a pas la valeurs positive qu’il possède habituellement ; il désigne de manière instable, selon les circonstances, 
plutôt des individus plus riches et plus instruits, ou plutôt diverses minorités, représentées par un vaste réseau d'associations ; mais bien souvent ces deux aspects se mêlent confusément chez les locuteurs-P. L'ajout de "globalisé » permet de souligner une facette importante de la divergence entre les deux points de vue : le sentiment qu'ont les locuteurs-P que ceux qui les rejettent appartiennent à un monde sans frontières, une sorte d'internationale pour laquelle les ancrages locaux seraient des obstacles à la promotion de valeurs humanistes. Dans une société où les médias jouent un rôle clé, la fracture discursive engendre chez ceux qui se voient en victimes un puissant ressentiment, qui ouvre aussi, comme on va le voir, un espace immense à l'exercice d'une parole protestataire à travers d'autres canaux.

Je propose de jeter un oil sur un texte qui me paraît révélateur de cette fracture discursive. Il s'agit d'un entretien (8 septembre 2018) avec une chercheuse nord-américaine, auteure d'un livre critique sur Trump. Il a été publié sur le site brésilien du journal espagnol El Pais, vraisemblablement parce qu'indirectement il concerne aussi Bolsonaro, qui à ce moment était candidat à la présidence du Brésil. Le titre de l'article, significativement, associe "débat démocratique » et " politiquement correct " pour les opposer à la " rhétorique " dangereuse des locuteurs-IM. L'emploi péjoratif de " rhétorique » est révélateur : il existe un bon usage du langage ("le débat démocratique ") et un usage déviant, " rhétorique ", celui des locuteurs-IM, qui ressortir à la tromperie et à la manipulation. 
Figure 1 - El País.

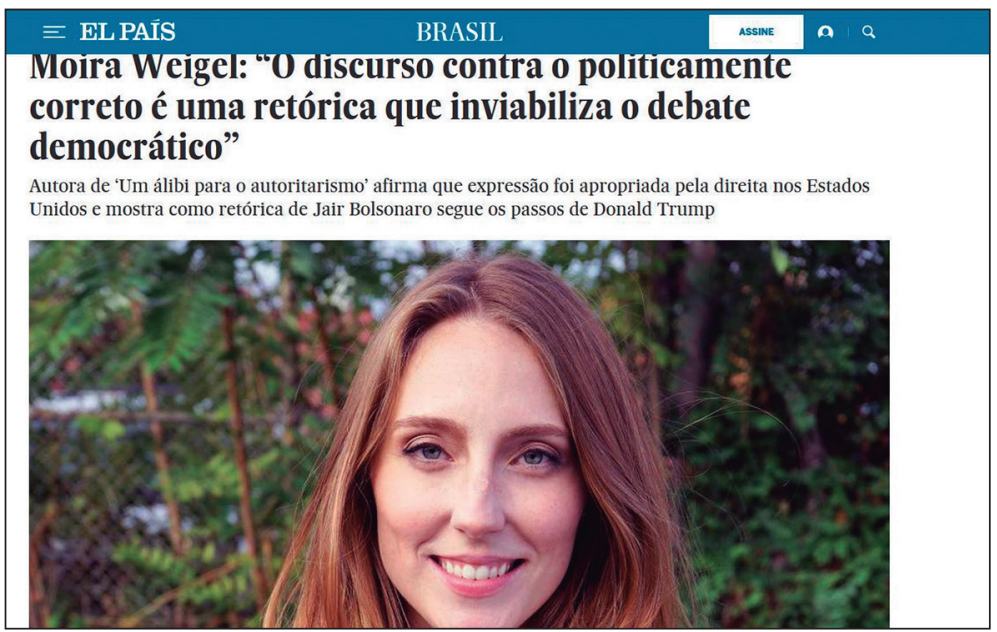

Source : El País ${ }^{2}$

Le site web où figure ce texte est celui d'El Pais, qui se présente comme « el periodico global ", publié en diverses langues. De fait, un tel entretien annule la frontière entre trois espaces (l'Espagne, les USA, le Brésil) ; il est présupposé qu'en tous lieux les élites partagent le même souci de défendre les valeurs " démocratiques ». Le choix de l'universitaire interviewée n'est pas indifférent non plus : une post-doctorante nord-américaine au croisement de l'étude des textes, du féminisme et de l'antiracisme, dont la recherche, de l'université de Cambridge (Angleterre) à celles de Yale et de Harvard (U.S.A.), participe des " gender studies ", de la théorie littéraire, des "media studies ", de la philosophie poststructuraliste, c'est-à-dire de l'interdiscours diffus qui sert d'appui, direct ou indirect, au politiquement correct ${ }^{3}$.

La convergence a priori des points de vue du journal El Pais et de la jeune chercheuse explique la manière dont se déroule l'interaction. Le journaliste ne discute pas les idées de $\mathrm{M}$. Weigel, il pose des questions brèves destinées à éclairer sa pensée. Au début de l'entretien celle-ci met obliquement en évidence la fracture discursive, en évoquant le rôle « fondamental » que joue chez Trump un usage « ultrajante » du langage :

\footnotetext{
2 Voir : https://brasil.elpais.com/brasil/2018/08/20/politica/1534788456_384604.html. Consulté le 20 mars 2020.

3 Son profil se trouve à l'adresse http://harvard.academia.edu/MoiraWeigel. Consulté le 1/2/2020.
} 
[...] a pesquisadora de Harvard detalha como a linguagem é parte fundamental da conexáo que Trump estabeleceu com seus seguidores. $\mathrm{O}$ mandatário americano, diz Weigel, recebe apoios justamente por dizer coisas "ultrajantes", consideradas inapropriadas pelas convençóes que estabelecem os limites do debate público. Cria empatia com parte expressiva da população porque "diz o que pensa" e por denunciar uma suposta conspiração de liberais com a imprensa, que teria o escuso objetivo de controlar inclusive as palavras que as pessoas comuns usam (WEIGEL, 2018, en ligne).

Cela permet au journaliste d'établir un lien avec Bolsonaro :

Náo escapa a um brasileiro que leia o texto de Weigel a memória das explosivas declaraçóes de Jair Bolsonaro, candidato à presidência da República (WEIGEL, 2018, en ligne).

M. Weigel propose une explication du phénomène :

Então, muito da dinâmica social ao redor do politicamente correto tem a ver com vergonha. Se você olha para os Estados Unidos, um país extremamente segregado racialmente, há muitas pessoas brancas, e que estão com sérias dificuldades financeiras, que ligam a tevê e veem uma pessoa rica dizendo que o jeito que elas falam é racista. E que elas deveriam aprender a falar como alguém que foi à universidade. Isso cria um sentido real de exclusão política e econômica, que se identifica com a narrativa do antipoliticamente correto. Então eu acho que a linguagem do Trump faz com que essas pessoas sintam menos vergonha do que elas dizem, porque ele próprio não tem nenhum pudor quando fala (WEIGEL, 2018, en ligne).

L'argumentation suit un schéma classique en sciences sociales, qui implique chez l'analyste une supériorité la fois morale et épistémique. Les dénonciateurs du politiquement correct seraient des " personnes blanches " habitées par une force négative, en l'occurrence le " racisme "; mais la cause, en dernière instance, serait économique : « sérias dificuldades financeiras » engendrées par une « crise global do capitalismo ». En filigrane, on retrouve l'exemplum privilégié d'un grand nombre d'argumentations sur le même thème, celui de l'Allemagne des années 1930 («ressurgimento desses movimentos de extrema direita»), où la 
crise économique a accru le racisme antisémite, affaibli la démocratie et favorisé l'arrivée des nazis au pouvoir.

E em meio à crise global do capitalismo que estamos vivendo, nós estamos vendo o ressurgimento desses movimentos de extrema direita e o aumento da sua popularidade porque eles falam para um público que sente ter sido excluído da história do progresso que deveria ter começado depois do fim da Guerra Fria (WEIGEL, 2018, en ligne).

Le problème est de savoir si cette analyse traditionnelle est suffisante, en d'autres termes si l'histoire se répète, d'une crise du capitalisme à l'autre. En réalité, les propos de M. Weigel ouvrent aussi la possibilité d'une lecture en termes de fracture discursive, comme le montre le passage cité plus haut :

[...] ligam a tevê e veem uma pessoa rica dizendo que o jeito que elas falam é racista. E que elas deveriam aprender a falar como alguém que foi à universidade. Isso cria um sentido real de exclusão política e econômica, que se identifica com a narrativa do antipoliticamente correto (WEIGEL, 2018, en ligne).

L'analyste, locutrice-M, cherche ici à restituer le point de vue-P, mais évidemment sans questionner la position de surplomb qu'implique son énonciation, celle que dénoncent précisément les locuteurs-P. C'est en se connectant à la Sphère autorisée (sous la forme de la télévision) que les locuteurs-IM entendraient dire par " un riche " (pour eux un locuteur-EG) que «o jeito que elas falam é racista» et que leur tort est de ne pas " falar como alguém que foi à universidade " (on notera l'instabilité, que nous avons évoquée plus haut, dans la caractérisation des élites : elle mêle richesse et niveau d'instruction). Cette conviction des locuteurs-IM est renvoyée par la chercheuse américaine à un " récit " sans fondement dans lequel certaines catégories de professionnels du langage ("professores», «imprensa») exclueraient les «pessoas comuns», en bas.

[...] essa narrativa passa uma ideia de que existe um pequeno grupo, formado por professores e pela própria imprensa, que está forçando essas mudanças de cima para baixo, às custas das 'pessoas comuns' (WEIGEL, 2018, en ligne). 
Cette disqualification des propos des locuteurs-IM est validée de manière performative par l'ethos de compétence qui ressort de l'analyse de leur comportement. La locutrice qui défend le point de vue-M montre qu'elle est capable d'expliquer à l'aide de schèmes issus des sciences sociales les erreurs dont sont coupables les locuteurs-IM, d'expliquer précisément pourquoi ils sont devenus des locuteurs-IM. Ces derniers apparaissent comme des sujets qui ne sont pas conscients des mécanismes qui les amènent à dévier moralement et intellectuellement, ils n'ont pas cette distance qui leur permettrait de comprendre que ceux qu'ils considèrent à tort comme des " riches " ou des " professeurs " qui les méprisent défendent en réalité les valeurs démocratiques universelles et éprouvent en fait de la compassion pour eux, puisque le vrai coupable est «a crise global do capitalismo».

On se trouve ainsi devant un système où chacun des deux termes se légitime par l'autre. Les locuteurs-M dénoncent ce qu'ils considèrent comme un usage immoral et cognitivement déficient du discours; pour défendre les valeurs de démocratie, d'ouverture, de respect d'autrui, etc., pour préserver la frontière qui nous sépare de forces obscures et menaçantes, il leur paraît vital d'analyser et de dénoncer les propos qu'ils jugent haineux et intellectuellement indigents. Les énoncés des locuteurs-P sont des objets d'analyse, et leurs auteurs ne sont malheureusement pas des gens avec qui il est possible de débattre en se conformant à des normes intellectuelles et morales.

Le problème est que les locuteurs-P voient dans leur condamnation et l'analyse de leurs énoncés une preuve de plus qu'ils sont exclus, incompris et méprisés par une minorité de privilégiés globalisée qui, pensant incarner le Bien, parlent avec autorité mais ignorent le vécu, les expériences, les difficultés des gens " de bon sens ", du " peuple », des " travailleurs », des " sans voix »... enracinés dans des cultures locales. Plus les locuteurs les plus légitimes de la Sphère autorisée, et parmi eux les chercheurs en sciences sociales, démontent et dénoncent les énoncés des locuteurs-P, plus ces derniers se sentent justifiés de rejeter ceux qui les rejettent et de jeter le discrédit sur l'ensemble des discours d'autorité.

On retrouve ici une manifestation de l'« interincompréhension " polémique (MAINGUENEAU, 1984) où chacun des adversaires traduit les énoncés de l'autre dans ses propres catégories. Il existe néanmoins une différence importante entre les polémiques classiques et cette situation de fracture discursive. Une polémique politique, littéraire, religieuse... oppose des adversaires qui partagent le même espace, le même régime de discours : les statuts des adversaires sont comparables et leurs productions textuelles aussi. Or la fracture discursive repose précisément sur une asymétrie entre d'une part des énonciations et des 
énonciateurs qui pensent que leur légitimité tient au fait qu'ils se soumettent à des normes intellectuelles, morales et textuelles adossées à des institutions sources d'autorité, et d'autre part des énonciateurs et des énonciations associés à des espace de faible légitimité, surtout les réseaux sociaux, où s'expriment des agrégats d'énonciateurs anonymes. Certes, il existe aussi dans la Sphère autorisée des locuteurs qui ne se conforment pas au point de vue- $M$, mais ils énoncent surtout dans des lieux périphériques et sont présentés par les locuteurs-P comme suspects : l'intérêt que suscitent parfois leurs propos auprès d'un vaste public est interprété comme la preuve qu'ils flattent les mauvais instincts de la multitude.

Ce n'est pas sans raison que je viens d'évoquer les réseaux sociaux; les nouvelles technologies de la communication jouent en effet un rôle essentiel dans cette asymétrie qui fonde la fracture discursive. Les locuteurs-P considèrent que s'ils investissent ces réseaux sociaux, c'est parce que la Sphère autorisée, contrôlée par les locuteurs-EG, leur est interdite. Pour les locuteurs-M, en revanche, c'est parce qu'ils sont dans l'incapacité de se soumettre aux normes intellectuelles et éthiques du " débat démocratique " que les locuteurs-P investissent les zones obscures de la communication digitale pour exprimer sans retenue leurs affects et répandre de fausses rumeurs.

Plutôt que de dire que les locuteurs-P « expriment » leurs idées en " utilisant " certaines possibilités que leur offre la communication digitale, il vaut mieux considérer - conformément aux présupposés de l'analyse du discours que les Sujets parlants sont indissociables des "moyens " de communication, autrement dit que ce ne sont pas des "moyens " : il n'existe pas d'une part des Sujets pleins, porteurs d'idées à exprimer, et d'autre part des instruments de communication pour les véhiculer. Les Sujets se construisent à travers les ressources qu'offrent les dispositifs d'énonciation disponibles en un lieu et à un moment donnés.

L'une des figures les plus connues des "Gilets jaunes ", Jérôme Rodrigues, a été blessé par un projectile de la police lors d'une manifestation, pendant qu'il diffusait en direct sur sa page Facebook.

Alors qu'il diffusait en direct des images de la fin du défilé à Paris, il a reçu un projectile. L'IGPN ${ }^{4}$ est saisie. C'est une vidéo impressionnante. Un gilet jaune qui participait ce samedi à la manifestation organisée à Paris entre le cours de Vincennes et la place de la Bastille a été atteint en plein visage par un projectile, alors même qu'il était en train de diffuser

${ }_{4}$ Il s'agit des services de police qui enquêtent sur la police. 
en direct sur Facebook des images des tensions entre les forces de l'ordre et les manifestants en fin de journée. Diffusée sur le compte Facebook de cet homme nommé Jérôme Rodrigues, la vidéo dure plus de onze minutes (RODRIGUES, 2019, en ligne). ${ }^{5}$

J. Rodrigues opère ici à la jointure entre deux espaces : d'une part celui de la manifestation à laquelle il participe, d'autre part l'espace sans bords des réseaux sociaux. Il n'a pas le statut d'un médiateur, d'un professionnel de l'information : il alimente directement sa page Facebook, sans filtre institutionnel. Sa vidéo se donne comme la " réalité », celle des gens " réels », " d'en bas », réprimés par des forces qui obéissent à des ordres venus d'en haut.

Les possibilités qu'offrent aux locuteurs-P les nouvelles technologies de la communication ne se limitent pas aux réseaux sociaux. Ils peuvent investir bien d'autres espaces. Par exemple les « commentaires » que les sites d'information permettent aux internautes de déposer en nombre illimité à la suite d'un article. Ce dispositif diffère considérablement de celui qui prévalait il y a encore quelques années dans la presse écrite, la radio et la télévision, où la parole du journaliste était sans appel : ils ne pouvaient pas publier leurs évaluations à côté des articles qu'ils lisaient.

Les textes publiés sur un site d'information relèvent de genres normés. Ils sont écrits par des journalistes et placés sous la responsabilité du directeur de la rédaction. Ils se conforment en outre à un positionnement déterminé dans le champ de la presse : positionnement professionnel (presse régionale ou nationale, spécialisée ou généraliste, etc.) et positionnement politique. Alors que les articles doivent poser un thème en se conformant à des normes de cohésion et de cohérence, les commentaires peuvent facilement devenir un espace de défoulement. Ils s'appuient seulement sur une relation prédicative au sens large : " je dis X à propos du texte $Y$ ». En outre, ils sont faiblement contraints : les affects s'expriment sans contrôle (on y trouve en particulier beaucoup de propos agressifs, voire injurieux) et les locuteurs ne se croient pas obligés d'élaborer des énoncés structurés, ni même de respecter l'orthographe ou la ponctuation. Ils ne sont pas soumis non plus aux contraintes qui s'imposent aux rédacteurs professionnels quant au référencement de leur texte par les moteurs de recherche, au choix de titres, d'intertitres, d'hyperliens. Cette divergence entre article et commentaires est étroitement liée à l'identité de leur source énonciative : ceux qui déposent des commentaires, à la différence des auteurs des articles, dotés d'un nom propre,

5 Disponible sur : https://www.midilibre.fr/2019/01/26/jerome-rodriges-figure-des-gilets-jaunes-gravement-blessea-loeil,7977404.php. Consulté le 23 mars 2020. 
forment des agrégats instables et éphémères de pseudonymes qui n’ont pas à répondre de ce qu'ils disent.

Dans un tel dispositif, la supériorité de la parole normée du journaliste est constamment mise en cause par son association avec un nombre indéfini d'énoncés à l'origine indéterminée qui l'utilisent comme d'appui. Il se produit un déplacement du centre de gravité : ce n'est plus l'article qui importe, mais la relation entre l'article et les commentaires qu'il suscite. Il arrive même souvent que les interactions entre producteurs de commentaires fassent oublier l'article qui les a suscités. Alors que le texte du journaliste implique une distance essentielle entre son auteur et ses lecteurs, la juxtaposition de commentaires fortement subjectifs crée une proximité entre commentateurs qui ne se connaissent pas mais qui dans le monde virtuel nouent et dénouent des complicités plus ou moins fugaces.

Le paradoxe sur lequel repose ce dispositif est que les commentaires bénéficient du vaste lectorat d'un site d'information reconnu, sans pour autant être soumis aux normes de la communication journalistique. Ils permettent d'exprimer ce qui auparavant aurait relevé de la sphère privée, voire de l'inavouable, mais qui se trouve sur un pied d'égalité avec le texte autorisé. Comme chaque commentaire est associé à des boutons qui permettent à l'internaute d'ouvrir sur des espaces connexes, en particulier Facebook ou Tweetter, il est même susceptible de toucher un public plus large, bien au-delà du site où il a été déposé.

Le terme de " commentaire " montre ainsi toute sa polyvalence. Le commentaire traditionnel porte sur des textes prestigieux, il implique une hiérarchie de valeur entre un texte d'autorité et une multitude d'énonciations qui cherchent à l'interpréter. Les commentaires qui nous intéressent ici tendent au contraire à occuper la position haute, à se poser en évaluateurs du texte commenté. Cette inversion de la hiérarchie peut faire songer à la catégorie du carnavalesque chez Bakhtine (1982). Mais le carnaval ne renverse symboliquement les hiérarchies instituées que pendant une période limitée ; les commentaires, eux, accompagnent de manière permanente les articles et s'accroissent même avec le temps. Même quand le commentaire approuve le point de vue défendu dans l'article, il le fait souvent selon des modalités qui ne relèvent pas du régime discursif adapté aux situations formelles, en activant une parole fortement subjective, voire brutale, qui est censée montrer un ethos d'authenticité.

D'un point de vue textuel aussi, ces commentaires présentent des particularités intéressantes. Habituellement, on distingue deux grands types d'organisation textuelle : a) celui de la conversation, où l'énoncé s'inscrit dans une chaîne d'interventions, avec des contraintes de divers ordres : en matière de 
ménagement des faces, d'ouverture et de clôture, de négociation des thèmes, d'enchaînement des énoncés... ; b) celui du genre de discours, qui assigne aux participants divers rôles au service d'une finalité reconnue, et qui implique une textualité planifiée préalable, plus ou moins connue à l'avance. Or les commentaires d'articles ne se soumettent ni aux règles de la conversation, ni à celles d'une textualité planifiée. Ils peuvent se réduire à un émoticône, une interjection, une phrase sans verbe, ou se présenter comme un long développement explicatif. Ils peuvent enchaîner sur un commentaire précédent, ou partir sans transition dans d'autres directions. Énoncés par nature seconds, réactifs et anonymes, ils n'ont pas à structurer un point de vue, à se rendre résistants à d'éventuelles contestations. Ils cherchent plutôt la connivence de ceux qui partagent le même point de vue ou, à l'inverse, l'affrontement avec des adversaires présents (l'auteur de l'article ou d'autres commentateurs) ou absents.

Ces caractéristiques sont indissociables du fait que la position de destinataire de ces commentaires est foncièrement problématique. On peut se demander si on n'a pas plutôt affaire à ce que j'ai proposé d'appeler (MAINGUENEAU, 2020) des sous-destinataires. Non pas un ou des individus identifiés, comme dans la conversation, ni les destinataires modèles impliqués par chaque genre de discours, lui-même ancré dans une scène englobante, mais un ensemble aux contours indéterminés, à la mesure d'énoncés qui se présentent comme la projection directe d'une subjectivité dont l'expression n'est pas régulée par la présence d'autrui ou le contrôle d'une institution. Ce " sous-destinataire " a pour corrélat un sous-locuteur : non seulement parce que la source du commentaire est pseudonyme et instable dans ses opinions et sa manière d'occuper l'espace du web, mais encore parce qu'il est largement affranchi des contraintes usuelles de la communication, de la responsabilité d'un Sujet parlant qui doit être cohérent et présentable, spécifié par une institution de parole et soumis au jugement d'un surdestinataire, au sens de M. Bakhtine (1984, p.336-337). L'auteur pseudonyme d'un commentaire n'est pas l'auteur pseudonyme d'une oeuvre littéraire.

Dans ces conditions, on comprend que les locuteurs-P exploitent intensément ce type de commentaire, qu'ils perçoivent comme un espace libéré du contrôle des locuteurs-P, un espace où ils peuvent sentir qu'ils ne sont pas seuls, qu'il existe une protestation diffuse contre ceux qu'ils jugent des oppresseurs illégitimes. Ce faisant, ils se confortent dans leur position de victimes d'un système qui les oblige à s'exprimer dans des lieux périphériques. Ainsi, les deux asymétries se répondent : celle entre les régimes $\mathrm{P}$ et $\mathrm{P}$, d'une part, et celle entre article et commentaires, d'autre part. Les commentaires d'articles ne sont pas plus des textes à proprement parler que les agrégats de locuteurs-P ne sont des 
partis, ni même des collectifs structurables. Dans les deux cas il y a asymétrie entre un espace structuré de positions soigneusement contrôlée (Sphère autorisée ou texte/genre de discours) et un espace foncièrement instable qui se soutient de la contestation du premier.

\section{Conclusion}

La notion de fracture discursive s'inscrit en français dans une matrice où figurent déjà des formules telles que "fracture sociale " et " fracture numérique ". La première dit l'opposition entre des élites qui seraient intégrées à un monde globalisé et les classes populaires qui tendraient à un repli identitaire ; la seconde désigne l'inégalité d'accès à la technologie numérique. Dans les deux cas il y a opposition entre deux niveaux dans la société : celui des privilégiés et celui des exclus. La fracture discursive est plus proche de la fracture sociale que de la fracture numérique, mais elle ne se laisse pas réduire à une opposition entre le local et le global ou entre le peuple et les élites, même si c'est à travers ces distinctions élémentaires qu'elle s'exprime souvent. La fracture discursive exclut des lieux de discours à l'autorité la plus forte, sur la base d'une déficience morale des locuteurs concernés. Cette déficience est perçue comme indissociable d'une déficience épistémique qui se traduit par un surinvestissement de canaux de communication incontrôlables et à large diffusion qui rendent possibles des modes d'énonciation non normés.

Une telle fracture n'est pas du même ordre que des clivages sociaux classiques (entre riches et pauvres, salariés et travailleurs indépendants, ouvriers et cadres, chômeurs et non chômeurs, etc.), mais les traverse tous dans des proportions variables. Elle ne se laisse pas non plus capter dans les positionnements politiques traditionnels. Certes, certains partis s'efforcent de s'ériger en porte-paroles des locuteurs-P, mais ces derniers répugnent à se reconnaittre dans les partis classiques. Ils vont plutôt adhérer à des individus qui, par leur usage atypique du discours, leur paraissent incarner une parole de vérité. À défaut d'un tel leader, ils peuvent apporter leurs votes à des partis dont le discours est essentiellement protestataire, avec tous les risques que cela comporte.

Pour l'analyste du discours cette situation est délicate. Il se trouve aux prise avec un objet qui devrait lui convenir, puisqu'il s'agit de réfléchir sur une fracture discursive. Mais, pour l'étudier, il doit s'interroger sur certains de ses présupposés.

Nul doute que dans leur grande majorité les analystes du discours partagent les valeurs du point de vue-M ; leurs convictions personnelles et la logique de leur appareil conceptuel et méthodologique les incitent à analyser les pro- 
pos de locuteurs-P comme foncièrement déficients. Le problème est que cette déficience est parfaitement prévisible, que l'enquête est toujours fructueuse. La question se pose de savoir si on peut se contenter de disqualifier les énoncés produits par les locuteurs-P, s'il ne faut pas réfléchir aussi sur la fracture discursive elle-même. Les chercheurs en sciences sociales et les locuteurs-M rapportent constamment les énoncés des locuteurs-P à des facteurs sociaux, mais ceux qui les analysent ainsi s'exceptent de l'univers du discours, considérant implicitement que leur soumission à des normes intellectuelles et morales partagées les place hors d'atteinte. Autrement dit, les propos des locuteurs-P s'expliqueraient socialement, mais le Vrai et le Bien s'expliqueraient eux-mêmes, ils ne relèveraient pas du discours.

Pourtant, la fracture discursive est un phénomène historique, un événement dans l'histoire de la distribution de la parole légitime. L'existence de locuteurs- $\mathrm{M}$ et la manière dont ils produisent et font circuler leurs énoncés ne peuvent échapper à l'ordre du discours. Ce déplacement du regard - des énoncés vers la fracture discursive - est cependant difficile à opérer. La fracture discursive n'est pas en effet un affrontement de positionnements, mais une configuration dont une foule d'acteurs participent et que personne ne contrôle. C'est une tâche importante que de réfléchir sur la reconfiguration de l'espace du discours qu'implique l'émergence d'une telle fracture discursive. Les locuteurs-M ont raison de dénoncer l'idée délirante qu'il existerait une sorte de complot contre " le peuple " menée par une minorité globalisée de privilégiés qui contrôleraient les médias, mais ce délire n'invalide pas la question sous-jacente, celle des conditions de possibilité de cette fracture.

La situation des analystes du discours est sur ce point plus inconfortable que celle des sciences dites "dures ", dont l'analyse est partagées entre des approches sociologiques qui cherchent à expliquer le fonctionnement et l'évolution des sciences par des facteurs " externes » et des approches épistémologiques qui cherchent à les expliquer par des facteurs " internes » aux sciences, étrangers donc aux " facteurs sociaux ». En effet, ces conceptions antagonistes de l'activité scientifique n'ont que très peu d'incidence sur le travail effectif des savants. Il n'en va pas de même en analyse du discours, et plus largement dans les sciences sociales, où la recherche dépend étroitement de la manière dont les chercheurs se représentent leur propre ancrage dans la société et les conflits qui la traversent.

En analysant rigoureusement les énoncés des locuteurs-P pour montrer leurs déficiences, les analystes du discours font, certes, oeuvres utile dans un monde familier et rassurant sur le plan éthique et épistémique, mais ils demeurent à l'intérieur de la fracture discursive. Le problème est que s'ils vont 
plus loin et s'interrogent sur cette fracture, ils peuvent avoir le sentiment qu'ils ouvrent la porte à des forces qu'ils contestent. C'est là une situation particulièrement difficile, puisque les deux termes de l'alternative - accepter la fracture et la contester - sont insatisfaisants. Autant dire que pour relever un tel défi intellectuel et moral, il faut des réponses complexes.

Une chose est sûre : l'instabilité que provoque l'interaction entre le discours politique traditionnel et la fracture discursive peut avoir des conséquences graves, comme le montrent le développement de mouvements sociaux incontrôlables et l'arrivée au pouvoir de dirigeants politiques irresponsables et/ou autoritaires.

\section{BIBLIOGRAPHIE}

BAKHTINE, M. Esthétique de la création verbale. Paris: Gallimard, 1984. [1979]. BAKHTINE, M. François Rabelais et la culture Populaire au Moyen Âge et sous la Renaissance. Paris: Gallimard, 1982. [1965].

MAINGUENEAU, D. Variaçóes sobre o ethos. São Paulo: Parábola, 2020.

MAINGUENEAU, D. Genèses du discours. Liège: Mardaga, 1984.

RODRIGUES, J. Jérôme Rodrigues, figure des "gilets jaunes», grièvement blessé à l'oeil. Midi Libre [En ligne], Jan. 2019. Disponible sur : https://www.midilibre. $\mathrm{fr} / 2019 / 01 / 26 /$ jerome-rodriges-figure-des-gilets-jaunes-gravement-blesse-aloeil,7977404.php. Consulté le 23 mars 2020.

WEIGEL, M. Moira Weigel: "O discurso contra o politicamente correto é uma retórica que inviabiliza o debate democrático". [Interview: Ricardo Della Coletta]. El País [En ligne], Sep. 2018. Disponible sur : https://brasil.elpais.com/ brasil/2018/08/20/politica/1534788456_384604.html. Consulté le 20 mars 2020. 